\title{
Effect of an Out-of-Sphericity Error on the Radial Stiffness of an Air Dynamic Bearing*
}

\author{
Woo Chun $\mathrm{CHOl}^{* *}$, Yong Ho SHIN*** \\ and Chung Whan $\mathrm{CHOI}^{* * * *}$
}

\begin{abstract}
Out $^{-}$of $^{-}$sphericity error is degree of deformation of an air bearing sphere deviated from a perfect sphere. This paper investigates numerically the effect of out-ofsphericity error on the radial stiffness of an air bearing. Three types of out-ofsphericity modes are considered. In this study, the stiffness is calculated from pressure distribution at the bearing surface, which is obtained by solving the Reynolds equation. In some cases, large out-of-sphericity errors are found to improve the stiffness of an air bearing. This implies that an air bearing of perfect hemispheres is not necessarily of the best performance. Thus, much labor and cost in manufacturing air bearings can be saved. In addition, the radial stiffness depends greatly on the force applying direction for bearings of radially deformed stator hemisphere.
\end{abstract}

Key Words : Out-of-Sphericity Error, Air Dynamic Bearing, Stiffness, Clearance, Wedge Effect, Eccentricity, Groove, Rotor, Stator

\section{Introduction}

Since an air dynamic bearing has an air layer between the stator and the rotor, it has less problems such as noise, heat generation and wear than a mechanical ball bearing. In addition, the air bearing is proper to support high speed rotating small bodies, and is expected to replace a mechanical bearing in laser printers, scanners, and hard disks.

A few researches have been reported on this type of air bearing. Saito et al. ${ }^{(1)}$ studied the relationship between the load and the radial stiffness in a gas lubricated spiral-grooved semi- ${ }^{-}$spherical bearing and its vibration characteristics. Czyzwewski et al. ${ }^{(2)}$ investigated the pressure distribution and performance of an eccentric gas journal bearing of finite length. Bootsma ${ }^{(3)}$ the distribution of pressure on a radially eccentric spiral-grooved semi spherical and conical bearings. Kawabata et al. ${ }^{(4)}$ studied the pumping effect of spiral-grooved Herringborne journal

* Received 24th April, 2000

** Department of Mechanical Engineering, Korea University, 1, Anam-dong, Sungbuk-ku, Seoul 136-701, Korea. E mail : wcchoi korea.ac.kr

*** Graduate School, Korea University

**** Hyundai Motors bearings. Murata et al. ${ }^{(5)}$ reported bearing manufacturing conditions having an effect on supporting load through applying the planar analysis theory on an eccentric spiral-grooved semi-spherical bearing. Park et al. ${ }^{(6)}$ and Choi et al. ${ }^{(7),(8)}$ studied spiral grooved air bearing performance.

This study investigates numerically the effect of an out of sphericity error on the radial stiffness of air bearings. The out of sphericity error is defined as a degree of deformation of a bearing hemisphere deviated from a perfect sphere. This effect of the out-of sphericity error has not been dealt with in other studies. The out of sphericity error is a very important parameter, since it can influence the performance and the manufacturing cost of an air bearing. In this study, the pressure distribution is obtained from the clearance distribution based on rotor position and outof-sphericity error. And, by integrating the pressure distribution over the air bearing area, the force applying to the rotor is obtained, and the stiffness is calculated from the force-displacement relation.

\section{Basic Theory}

\subsection{Governing equation}

Figure 1(a) shows a schematic of the air dynamic bearing dealt with in this study. This bearing 


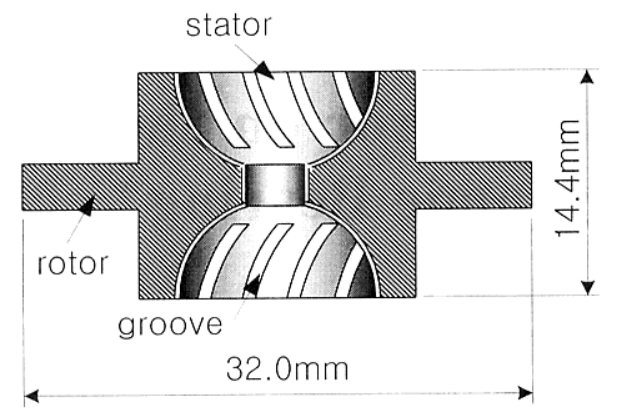

(a) Stator and rotor

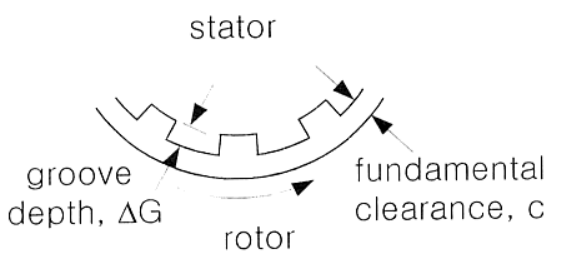

(b) Groove depth and fundamental clearance

Fig. 1 Schematic of a hemispherical spiral-grooved air bearing

is a self-acting spiral-grooved hemispherical air bearing. As the rotor starts to rotate, the environmental air flows into the gap between the rotor and the stator, and the pressure rises due to the wedge effect, supporting the rotor weight. Figure $1(b)$ shows the design variables of the air bearing.

The air bearing supports the rotor using air lubrication. Thus, the Reynolds equation is used as the governing equation.

$$
\begin{aligned}
& \frac{\partial}{\partial x}\left(h^{3} \frac{\partial p^{2}}{\partial x}\right)+\frac{\partial}{\partial y}\left(h^{3} \frac{\partial p^{2}}{\partial y}\right) \\
& \quad=12 \mu\left\{U \frac{\partial(p h)}{\partial x}+V \frac{\partial(p h)}{\partial y}+2 \frac{\partial(p h)}{\partial t}\right\}
\end{aligned}
$$

where $x$ and $y$ are coordinates, $h$ is clearance, $p$ is pressure, and $\mu$ is dynamic viscosity.

The equation is simplified and non-dimensionalized by applying the following conditions.

$$
V=V(\bar{X}), \quad U=0, \quad \frac{\partial}{\partial t}=0 \quad \text { (steady state) }
$$

In order to place nodal points along the grooves regardless of the groove angle, coordinate transformations are done. Coordinate transformations are conducted two times to make grooves perpendicular to the circumference of the stator as shown in Fig. 2(c) and finally the $x^{\prime \prime}-y^{\prime \prime}$ coordinate system is obtained. The non-dimensionalized governing equation is rewritten based on the $x^{\prime \prime}-y^{\prime \prime}$ coordinate system using Jacobians, and transformed into algebraic equations according to a finite difference method. The details can be found in the Ref. ( 8 ). With boundary condi-

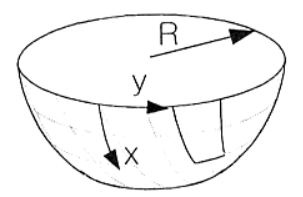

(a) Bearing surface

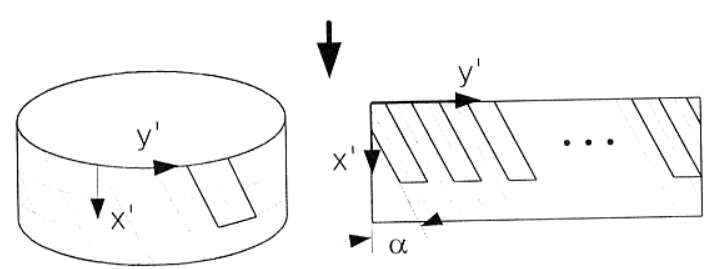

(b) Transformed plane (slanted grooves)

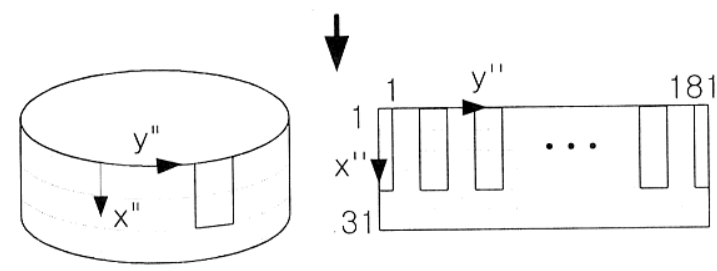

(c) Transformed plane (straight grooves)

Fig. 2 Coordinate systems

tions, the equations are solved using TDMA (TriDiagonal Matrix Algorithm) $)^{(9)}$ and circular TDMA ${ }^{(10)}$ for the pressure distribution at the gap.

In this study, the design parameters are set as follows :

- Fundamental clearance : $c=4 \mu \mathrm{m}$

- Groove depth : $\Delta G=4 \mu \mathrm{m}$

- Rotational speed : $\omega=18000 \mathrm{rpm}=1885 \mathrm{rad} / \mathrm{s}$

- Bearing radius : $R=6.2 \times 10^{-3} \mathrm{~m}$

- Bearing number : $\Lambda=6 \frac{\mu \omega}{p_{a}}\left(\frac{R}{c}\right)^{2}=4.789$

In this study, the number of nodal points is 31 and 181 in the $x^{\prime \prime}$ and the $y^{\prime \prime}$ direction, respectively.

\subsection{Stiffness of the air bearing}

In the previous experimental study ${ }^{(6)}$, the force applied to the rotor was found to be linear to the displacement of the rotor for small rotor displacements, and became nonlinear for large displacements. In this study, the stiffnesses of an air bearing are defined as follows.

$$
\left[\begin{array}{l}
\Delta f_{x} \\
\Delta f_{y}
\end{array}\right]=\left[\begin{array}{ll}
K_{x x} & K_{x y} \\
K_{y x} & K_{y y}
\end{array}\right]\left[\begin{array}{c}
\Delta x \\
\Delta y
\end{array}\right]
$$

The main stiffness term $K_{m}\left(=K_{x x}=K_{y y}\right)$ and the cross $^{-}$coupled stiffness term $K_{c}\left(=-K_{x y}=K_{y x}\right)$ are calculated from Eq. ( 3 ).

In this numerical analysis, rotor displacement (or eccentricity) is an input, from which the pressure distribution is obtained. The pressure distribution is 
integrated over the area of the stator surface, and the restoring force is calculated. The stiffness is obtained from the force-displacement relation.

\section{Out-of-Sphericity Error}

An out ${ }^{-}$of $^{-}$sphericity error is defined as a degree of deformation of an air bearing stator hemisphere from a perfect sphere. If the out- ${ }^{-}{ }^{-}$sphericity error is zero (perfect sphere), the clearance is uniform and the same as the fundamental clearance when the rotor is located at an uneccentric position. If the out-ofsphericity error is nonzero, the clearance distribution is not uniform. This uneven distribution affects the pressure as well as the stiffness and the load capacity of the air bearing.

A stator hemisphere can be deformed in many ways depending on manufacturing conditions. In this study, three types of out-of-sphericity modes are considered. Firstly, the hemisphere is enlarged axially and shortened radially (mode 1), as shown in Fig. 3 ( a ). In this figure, the out-of-sphericity is denoted by a. Secondly, the hemisphere is enlarged radially and shortened axially (mode 2), as shown in Fig. 3(b). Lastly, the hemisphere is deformed radially to have an elliptic cross $^{-}$section (mode 3). For the three modes, the clearance distributions are determined as follows.

- mode 1: $h=h_{p s}-a \cos \left(\pi x^{\prime \prime} / x^{\prime \prime}{ }_{\text {max }}\right)$

- mode 2: $h=h_{p s}+a \cos \left(\pi x^{\prime \prime} / x^{\prime \prime}{ }_{\text {max }}\right)$

- mode $3: h=h_{p s}-a \cos \left(2 \pi y^{\prime \prime} / y^{\prime \prime}{ }_{\text {max }}\right)$

where $h_{p s}$ is the clearance distribution for a perfect sphere (' 0 ' out-of-sphericity error).

\section{Results and Discussion}

\section{1 Pressure and clearance distributions}

For the 3 modes, clearance and pressure distributions are compared when the rotor is located at an uneccentric position. Only the distributions at the upper hemisphere are considered, because the bottom hemisphere has the same distributions as the upper one. In a spiral-grooved air-dynamic bearing, the pressure rises due to the wedge effect, and increases as the clearance decreases.

Figure 4 shows the clearance and the pressure for mode 1 , when the out ${ }^{-}$of $^{-}$sphericity error is $1 \mu \mathrm{m}$. The pressure is low near the upper region (small $x^{\prime \prime}$ node number) of the upper hemisphere and high at the lower region. This is because of the clearance distribution. The overall pressure is relatively low.

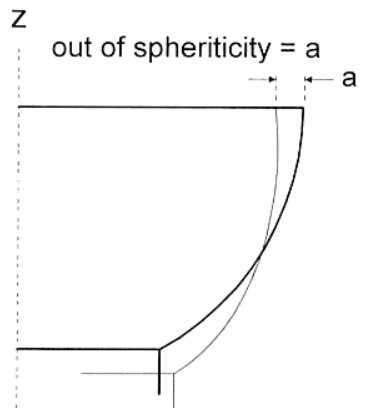

( a ) Mode 1

Z

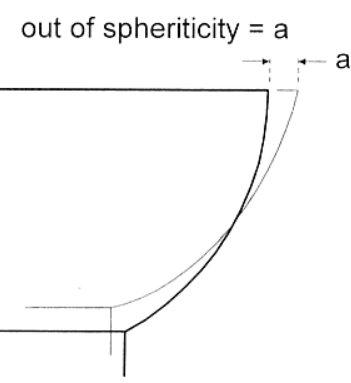

(b) Mode 2

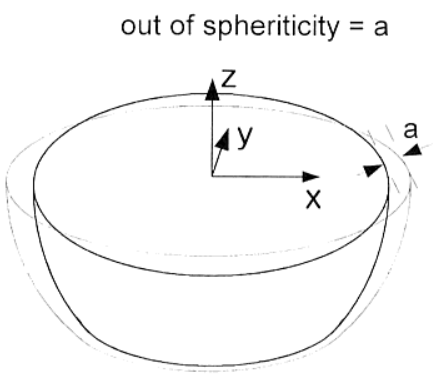

(c) Mode 3

Fig. 3 Modes of the out ${ }^{-}$of $^{-}$sphericity error

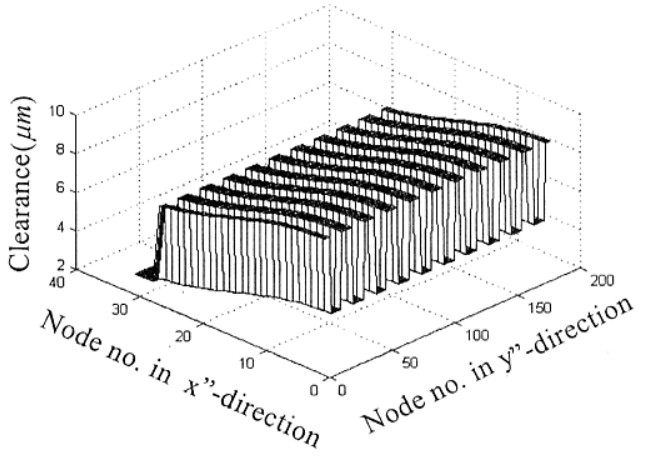

(a) Clearance distribution

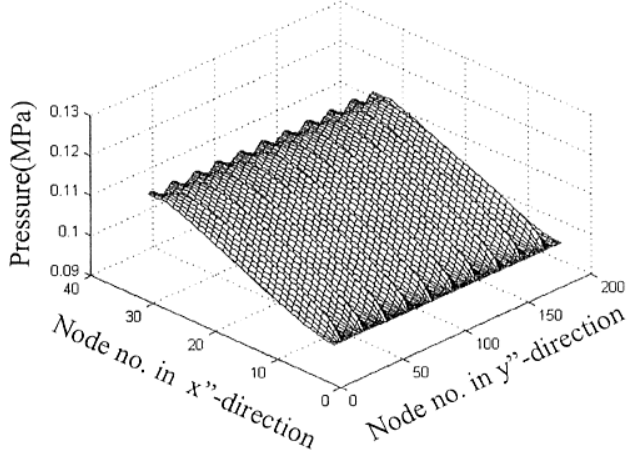

( b ) Pressure distribution

Fig. 4 Clearance and pressure distribution of mode 1 


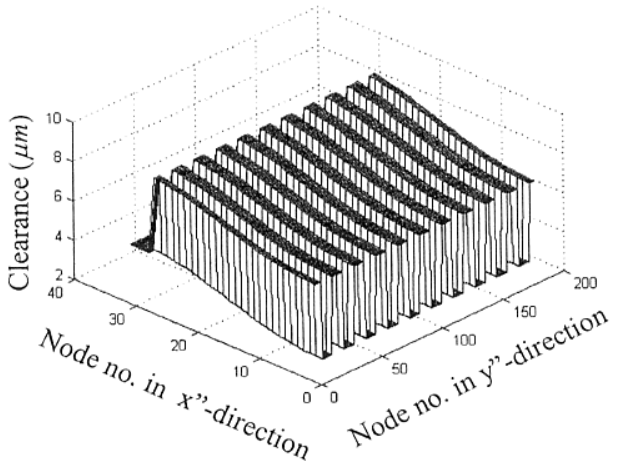

(a) Clearance distribution

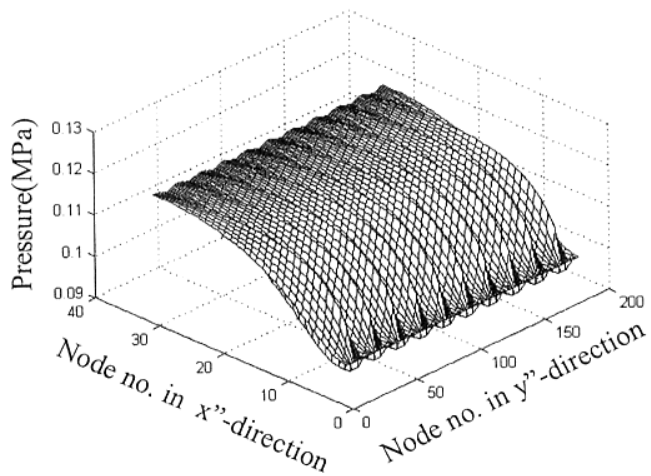

(b) Pressure distribution

Fig. 5 Clearance and pressure distribution of mode 2

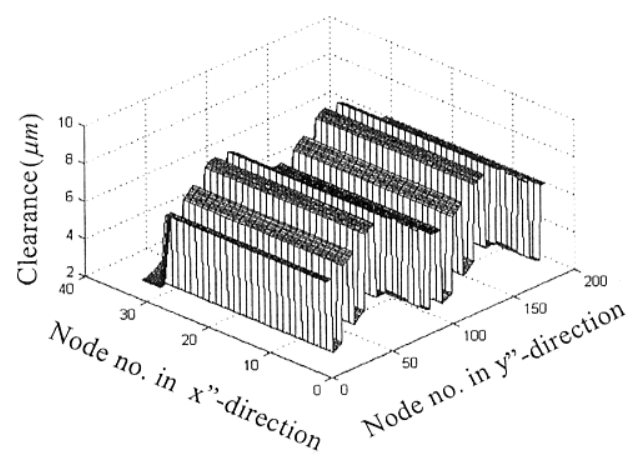

(a) Clearance distribution

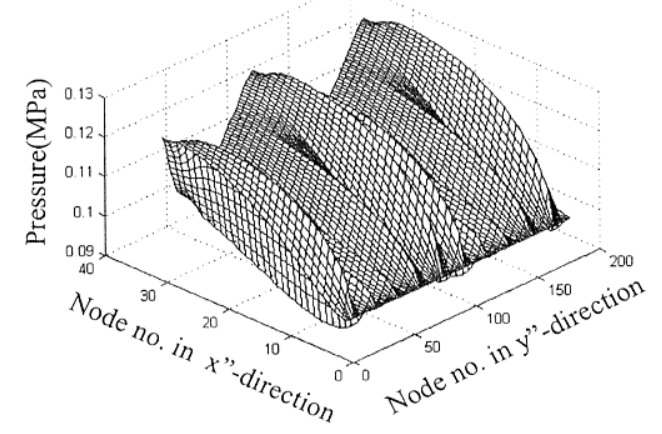

(b) Pressure distribution

Fig. 6 Clearance and pressure distribution of mode 3

Figure 5 shows the clearance and the pressure for mode 2. The pressure is much higher near the upper region due to small clearance than that for mode 1. The overall pressure is also higher than that for mode 1.

Figure 6 shows the distributions for mode 3 , where the hemisphere is enlarged in the $X$ direction and shortened in the $Y$ direction. The pressure is high in the $X$ direction due to the small clearance, and is low in the $Y$ direction due to large clearance.

\section{2 Stiffness according to the out-of-sphericity error}

The effect of the out- ${ }^{-}{ }^{-}$sphericity error on the radial stiffness is investigated for the 3 modes, as the out $^{-}$of $^{-}$sphericity error is increased from 0 to $1 \mu \mathrm{m}$. Because of the bearing shape, the pressure at the upper region contributes to force mainly in the radial direction, while the pressure at the lower region in the axial direction. Thus, the pressure increase at the upper region enhances the radial stiffness.

Figure 7 shows the stiffness for modes 1 and 2 with respect to the out ${ }^{-}$of $^{-}$sphericity error. The stiffness for mode 1 decreases as the out ${ }^{-}$of $^{-}$-sphericity error increases. This is because, as the out-of-spher-

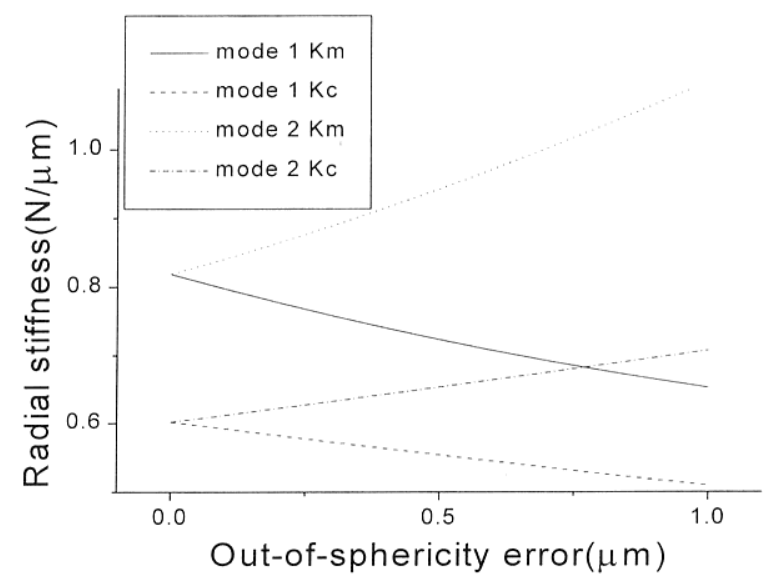

Fig. 7 Radial stiffness for modes 1 and 2

icity error increases, the clearance also gets large and the pressure decreases. For mode 2 , as the out-ofsphericity error increases, the pressure becomes high at the upper region because of the clearance decrease.

Mode 1 is undesirable if the bearing is used for a radial support, because the radial stiffness becomes small due to the out ${ }^{-}$of-sphericity error. The stiffness for mode 2 increases as the out-of-sphericity error 


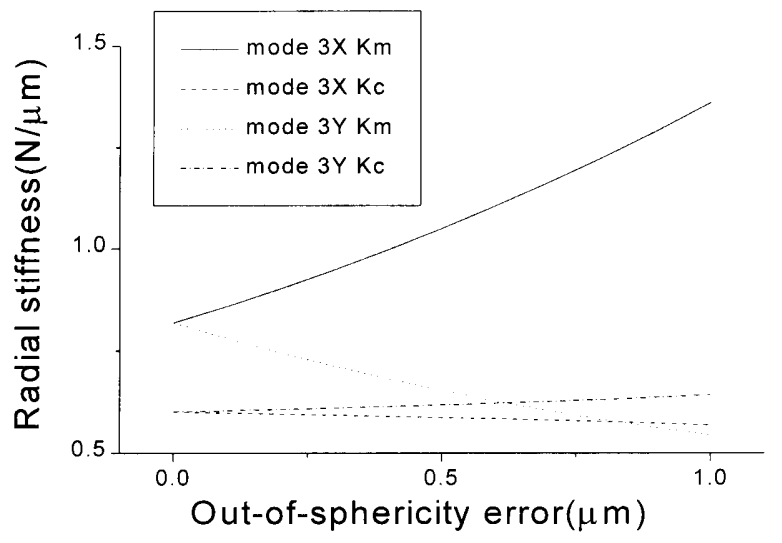

Fig. 8 Radial stiffness for mode 3

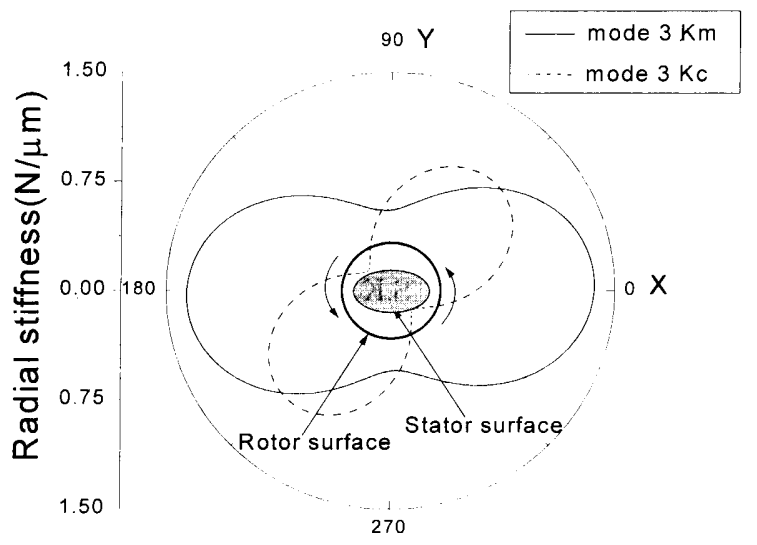

Fig. 9 Radial stiffness for force application direction increases. This means that a deformed hemisphere has better stiffness than a perfect hemisphere. This is important because it is not necessary in some cases to make a bearing hemisphere perfectly so that the manufacturing cost and labor can be reduced. A similar result was obtained in the experimental study $^{(6)}$. However, such air bearings can become unstable in the presence of vibration and impulse due to a very small local clearance, when the out-ofsphericity error is too large.

Figure 8 shows the stiffness with respect to the out ${ }^{-}$of-sphericity error for mode 3 . The bearing hemisphere is enlarged in the $X$ direction and shortened in the $Y$ direction. In mode $3, K_{m}$ and $K_{c}$ are varying according to the displacement applying direction due to elliptic radial cross section, which means that $K_{x x}$ and $K_{y y}$ are not the same and $-K_{x y}$ and $K_{y x}$ are not the same. This means that the radial stiffness terms for the mode 3 are determined by the displacement applying direction. Modes $3 X$ and $3 Y$ imply that the displacement applying directions are $X$ and $Y$ directions, respectively. As the out-of-sphericity error increases, the stiffness also becomes large in the $X$ direction, but decreases in the $Y$ direction. These are because of the clearance distribution. In addition, mode $3 X$ has much larger stiffness than mode $3 Y$.

Figure 9 shows the radial stiffness for mode 3 for various force applying directions in a polar coordinate frame, when the out-of-sphericity error is $1 \mu \mathrm{m}$. The stiffness is shown to depend greatly on the force applying direction. It has a maximum value in the $X$ axis, and a minimum value in $Y$ direction, but the values don't coincide with axes. This is because of the wedge effect which deviates the force application direction from eccentricity applied axis and an outof-sphericity error. And there exist regions where stiffnesses are very small in the $y$ direction. The force applying direction is very important, when using an air bearing of mode 3 .

\section{Conclusions}

This study investigates the effect of an out-ofsphericity error of a stator hemisphere on the radial stiffness. Three types of out $^{-}$of $^{-}$sphericity modes are considered. As a result, conclusions are made as follows :

- When a stator hemisphere is enlarged axially and shorten radially, the radial stiffness becomes small as the out-of-sphericity error increases.

- When a stator hemisphere is shortened axially and enlarged radially, the radial stiffness becomes large, as the out-of-sphericity error increases. However, the air bearing becomes very unstable to vibration or impulse due to a very small local clearance if the out-of-sphericity error is too large.

- When a stator hemisphere is deformed radially, the radial stiffness depends significantly on the force applying direction.

When an air bearing of an out-of-sphericity error is properly utilized, better performance can be achieved, with saving manufacturing cost and time.

\section{References}

(1) Saito, Y., Makino, H. and Tamura, A., Characteristics of Gas-Lubricated Spherical Spiral Groove Bearings, Journal of JSLE, Vol. 28, No. 3 (1983), pp. 221-227.

(2) Czyzwewski, T. and Titus, P., Analysis of an Arbitrarily Misaligned Self-Acting Gas Film Journal Bearing of Finite Length, Wear, Vol. 114 (1987), pp. 367-379.

(3) Bootsma, J., Spherical and Conical Spiral Groove Bearings, ASME J. of Lubrication Technology, (1975), pp. 236-249.

(4) Kawabata, N., Ashino, I., Sekizawa, M. and Yamazaki, S., Spiral Grooved Bearing Utilizing the Pumping Effect of a Herringbone Journal Bear- 
ing, JSME Int. J., Ser. III, Vol. 34, No.3 (1991), pp. 411-418.

(5) Murata, S., Miyake, Y. and Kawabata, N., Exact Two-Dimensional Theory of Spherical Spiral Groove Bearings, ASME J. of Lubrication Technology, Vol. 102 (1980).

(6) Park, K.H., Choi, J.H., Choi, W.C., Kim, K., Woo, K.M. and Kim, S.K., Measurement of Radial Performance in Spiral-Grooved Air-Dynamic Bearing, KSPE, Vol. 16, No. 2 (1999), pp. 23-30.

( 7 ) Choi, W.C., Park, K.H., Choi, J.H., Kim, K. and Koh, B.C., Study on the Performance of SpiralGrooved Semi-Spherical Air Bearings Using
Taguchi Method, JSME Int. J., Ser. C, Vol. 43, No. $1(2000)$.

(8) Choi, W.C., Shin, Y.H. and Choi, J.H., Analysis of Axial Load Characteristics of Air- Dynamic Bearings of Various Curvatures, J. KSPE, Vol. 17, No. 3 (2000), pp. 129-135.

(9) Patankar, S., Numerical Heat Transfer and Fluid Flow, (1980), pp. 52-54, McGraw-Hill.

(10) Patankar, S. and Sparrow, E., Fully developed Flow and Heat Transfer in Duct Having Streamwise-Periodic Variations of Cross $^{-}$sectional Area, Trans. of the ASME, Vol. 99 (1977), pp. 180-186. 